\title{
Profil spermiologique de l'époux dans les couples infertiles en milieu négro-africain au Sénégal
}

\author{
Babacar DIAO1, Oumar FAYE2, Papa Ahmed FALL1, Abdoulaye Séga DIALLO2, \\ Alain Khassim NDOYE1, José Marie AFOUTOU2
}

1 Service d'Urologie -Andrologie, Centre Hospitalier Universitaire Aristide Le Dantec, Dakar, Fann-Sénégal

2 Laboratoire de Cytogénétique et Biologie de la Reproduction, Centre Hospitalier Universitaire Aristide Le Dantec, Dakar, Sénégal

\section{RESUME}

Objectif: Préciser la part de responsabilité de l'homme dans l'infertilité du couple en milieu négro-africain au Sénégal et établir son profil spermiologique.

Matériel et Méthode : Nous nous sommes intéressés à l'exploitation de 17459 spermogrammes et 5563 tests post-coïtaux de patients suivis pour infertilité primaire et secondaire du couple entre janvier 1982 et décembre 2002. Les spermogrammes des tests post-coïtaux négatifs et déficients ont été étudiés afin de mettre en évidence la responsabilité masculine. Concernant les spermogrammes, nous avons étudié l'âge des patients, le mode de prélèvement, le volume de l'éjaculât, les caractéristiques spermiologiques.

Résultats : II y avait deux fois plus de stérilité primaire $(68,4 \%)$ que de stérilité secondaire $(31,6 \%)$. La responsabilité masculine $(31,7 \%)$ était deux fois plus importante que celle de la femme $(14,7 \%)$. Vingt huit pour cent des patients présentaient une hypospermie. Une oligozoospermie isolée était notée $10 \%$ des cas et une azoospermie était retrouvée dans $23 \%$ des cas. Les altérations spermiologiques qualitatives étaient observées dans $44,3 \%$ des cas. Une spermoculture positive était notée dans $21,3 \%$ des cas. Les associations (altérations spermiologiques qualitatives et anomalies de nombre) ont été observées dans $43,4 \%$ des cas. L'oligo-asthéno-tératozoospermie (OATS) avec spermatozoïdes à tête allongée, restes cytoplasmiques et angulation, caractéristique de la varicocèle a été noté de façon significative chez des porteurs de varicocèle ( $97 \%$ des porteurs de varicocèle droite et $98,2 \%$ des cas de varicocèle bilatérale). La dystrophie réactionnelle épididymo-sémino-prostatique avait été notée dans $11,9 \%$ des cas

Conc/usion : La part de responsabilité masculine est bien réelle. Le profil spermiologique de l'époux des couples stériles dans notre contexte est caractérisé par l'importance des altérations polymorphes comme l'oligoasthéno-térato-nécrozoospermie et de l'azoospermie sécrétoire.

Mots clés : infertilité masculine, oligo-asthénotératozoospermie, azoospermie sécrétoire

Correspondance :

Dr Alain Khassim NDOYE - CHU Aristide Le Dantec, BP 5321, Dakar, Fann-Sénégal - Email alainndoye@hotmail.com 


\section{INTRODUCTION}

La femme a longtemps été considérée comme la seule ou tout au moins la principale responsable de l'infertilité conjugale. De nombreuses femmes ont été et demeurent marginalisées voire répudiées du fait de cette confusion liée à l'ignorance des données éthiopathogéniques de l'infertilité conjugale. Cependant depuis quelques décennies, les progrès de la biologie de la reproduction ont établi que les responsabilités soient partagées dans la stérilité du couple. L'objectif de notre travail est de préciser la part de responsabilité de l'homme dans l'infertilité du couple en milieu négro-africain et d'établir son profil spermiologique.

\section{MATERIEL ET METHODE}

II s'agit d'une étude rétrospective qui s'est intéressée à l'exploitation de spermogrammes et tests post-coïtaux (de Hünher) de patients suivis pour infertilité primaire et secondaire du couple entre janvier 1982 et décembre 2002. Etaient inclus dans cette étude les spermogrammes de patients mariés quel que soit leur âge. Les spermogrammes des célibataires ont été exclus de notre étude.

Pour les tests post-coïtaux il s'agissait du test post coïtal. Ce test était pratiqué entre 4 à 8 heures après un rapport sexuel en période pré-ovulatoire (24 à 48 heures avant le décalage thermique) avec une abstinence sexuelle de 3 à 5 jours recommandée au couple. La patiente devait rester allongée 30 à 60 minutes après le coït. Un prélèvement de mucus endocervical était effectué. Nous nous sommes intéressés à l'analyse de la glaire, aux éléments spermatiques et aux résultats en fonction du type d'infertilité. Le test était jugé négatif s'il n'y avait pas de spermatozoïde, déficient s'il montrait des spermatozoïdes immobiles ou mobiles sur place, positif s'il y avait des spermatozoïdes en progression linéaire rapide. Les spermogrammes des tests post-coïtaux négatifs et déficients ont été étudiés afin de mettre en évidence la responsabilité masculine.

Concernant les spermogrammes, les méthodes de recueil et d'analyses étaient réalisées conformément aux recommandations de l'OMS [3]. Le mode de prélèvement était un coït interrompu dans $91,3 \%$ des cas et une masturbation dans $8,7 \%$ des cas. Nous avons étudié l'âge des patients, le volume de l'éjaculât (normal entre 2 et $6 \mathrm{ml}$, hypospermie si le volume était inférieur à 2 $\mathrm{ml}$, hyperspermie si le volume était supérieur à $6 \mathrm{ml}$ ) et les caractéristiques spermiologiques:

- Numération des spermatozoïdes : elle était considérée comme normale si le nombre de spermatozoïdes par $\mathrm{ml}$ était compris entre 20 et 80 millions et le nombre total entre 60 et 200 millions. II y avait oligozoospermie si la numération unitaire était inférieure à 20 millions par $\mathrm{ml}$. La polyzoospermie était retenue lorsque la numération unitaire était supérieure ou égale à 80 millions par $\mathrm{ml}$. Le diagnostic d'azoospermie était posé lorsqu'il y avait une absence totale de spermatozoïdes dans l'éjaculât. Cette azoospermie était qualifiée de sécrétoire en présence d'un taux de FSH élevé supérieur à deux fois la normale.

- Mobilité : l'asthénospermie était définie par un taux de spermatozoïdes mobiles inférieur à $50 \%$.

- Vitalité : la nécrozoospermie était retenue quand il y avait plus de $25 \%$ de spermatozoïdes morts initialement.

- Morphologie : la tératozoospermie était retenue quand le taux de spermatozoïdes présentant une morphologie anormale était supérieur à $70 \%$.

- La "fécondance du sperme" a été évaluée à partir de : la numération, la mobilité, la morphologie et la vitalité des spermatozoïdes. Une baisse de la fécondité était retenue si la numération unitaire était inférieure à 5 millions $/ \mathrm{ml}$, la mobilité et la morphologie normales inférieures à $20 \%$, la vitalité inférieure à $50 \%$.

- L'existence d'une infection spermatique ou spermoculture positive : la spermoculture était considérée comme positive lorsqu'il existe un germe banal à une concentration supérieure à $1000 \mathrm{CFU} / \mathrm{ml}$, ou lorsqu'un germe franchement pathogène est retrouvé quelle que soit sa concentration, le recueil du sperme étant pratiqué après miction.

L'exploitation des dossiers en Urologie a permis de préciser les antécédents médicaux et les résultats de l'examen physique des patients:

- notion d'infection uro-génitale, de torsion du cordon spermatique ;

- existence d'une varicocèle, dont le diagnostic était posé à la mancuvre de Valsalva et confirmé par l'échographie doppler, d'un testicule non descendu, d'une hypotrophie ou d'une atrophie testiculaire.

Ces données ont été exploitées à partir logiciel EPI INFO version 6 .

\section{RESULTATS}

L'analyse des tests post-coïtaux et des analyses spermiologiques de contrôle des tests post-coïtaux négatifs et déficients $(n=3411)$ a révélé que dans $48,9 \%$ des cas, malgré une glaire normale, il y avait une 
infertilité. II y avait deux fois plus d'infertilité primaire $(68,4 \%)$ que d'infertilité secondaire $(31,6 \%)$. La responsabilité masculine $(31,7 \%)$ était deux fois plus importante que celle de la femme $(14,7 \%)$. La responsabilité était double dans $53,6 \%$ des cas.

Concernant l'analyse des 17459 spermocytogrammes, la moyenne d'âge observée chez nos patients était de 33,2 ans avec des extrêmes de 19 ans et 65 ans. Les antécédents médicaux et les anomalies retrouvés dans les dossiers des patients sont rapportés au Tableau 1.

Vingt huit pour cent des patients présentaient une hypospermie (Figure 1). A la numération de spermatozoïdes, une oligozoospermie était notée dans $46,5 \%$ des cas mais elle n'était isolée que dans $10 \%$ des cas ; une azoospermie était retrouvée dans $23 \%$ des cas (Figure 2). La forme sécrétoire représentait $71 \%$ de l'ensemble des cas d'azoospermie. Les altérations spermiologiques qualitatives, rapportées sur la Figure 3 , étaient observées dans $44,3 \%$ des cas. Une spermoculture positive était notée dans $21,3 \%$ des cas. Les associations (altérations spermiologiques qualitatives et quantitatives) ont été notées dans $43,4 \%$ des cas (Figure 4).

Une baisse de la "fécondance du sperme" était observée $92,7 \%$ des cas. L'oligo-asthéno-tératozoospermie (OATS) avec spermatozoïdes à tête allongée, restes cytoplasmiques et angulation, caractéristique de la varicocèle, a été notée de façon significative $(p<0,0001)$ chez des porteurs de varicocèle ( $97 \%$ des porteurs de varicocèle droite et $98,2 \%$ des cas de varicocèle bilatérale).

La dystrophie réactionnelle épididymo-séminoprostatique (caractérisée par une oligo-asthénotératozoospermie avec plus de $5 \%$ de spermatozoïdes à flagelles enroulées) avait été notée dans $11,9 \%$ des cas. La spermoculture pratiquée chez les patients présentant une dystrophie réactionnelle épididymosémino-prostatique était positive dans $66,8 \%$ des cas.

\section{DISCUSSION}

Concernant la responsabilité masculine de l'infertilité de couple, elle pose le problème fondamental de la reconversion des croyances et des mentalités dans une société où une femme sans enfant est généralement culpabilisée. Cette responsabilité masculine a déjà été rapportée par d'autres auteurs [2, 7, 8].

L'âge moyen de nos patients confirme les résultats de Afoutou et al. [2] et recoupe les données de la littérature internationale. En effet une tranche d'âge de 25 à 34 ans a été rapportée par Ikéchebelu et al. [8] au Nigéria, chez $67,2 \%$ des patients de leur série et une moyenne d'âge de 29 ans est retrouvée en France [4]. Cela pourrait s'expliquer par le fait que durant cette période le désir de paternité est intense poussant les jeunes mariés qui n'arrivent pas à procréer à se confier plus rapidement à un médecin ; au delà de cet âge le désespoir et le fatalisme prennent le dessus.

Quant au volume du sperme, il traduirait essentiellement la capacité sécrétoire des glandes séminales [5]. Cependant le taux d'hypospermie retrouvé dans notre série devrait être interprété à la lumière du mode de prélèvement. Le coït interrompu a été le plus fréquent dans notre étude car la masturbation est difficilement acceptée dans notre société et entraîne souvent un blocage empêchant le prélèvement. Avec ce mode de prélèvement, il existe un risque important d'erreurs par perte de la partie initiale de l'éjaculât (la plus concentrée en spermatozoïdes) ou par contamination par les sécrétions vaginales. Cependant, un certain nombre d'éléments peuvent influer sur le volume de l'éjaculât parmi lesquels le degré d'excitation et le délai d'abstinence.

Concernant les anomalies de la numération des spermatozoïdes, le taux d'oligozoospermie isolée retrouvé dans notre série rentre dans la fourchette des taux rapportés en Ethiopie [9] et au Mexique [11], respectivement de $11 \%$ et $7 \%$. Guérin [5] a rapporté que l'oligozoospermie pourrait être liée dans certains cas à une hyperspermie ou à une polyspermie. II s'agirait d'une oligozoospermie relative dite de dilution, car le nombre total de spermatozoïdes dans l'éjaculât n'est pas abaissé. De tels cas n'ont pas été retrouvés dans notre étude où chez certains patients, la polyspermie permettait de compenser une oligozoospermie unitaire.

La prévalence de l'azoospermie dans notre étude est proche de celle de Afoutou et al. [2] qui était de $25 \%$, avec prédominance de l'azoospermie sécrétoire. $\mathrm{Ce}$ constat, associé à la prédominance l'infertilité primaire par rapport à l'infertilité secondaire, nous laisse croire que l'influence des troubles endocriniens, malformatifs et génétiques sur la reproduction en milieu négro-africain serait plus marquée que celle des dystrophies inflammatoire et/ou infectieuses.

Les altérations spermiologiques qualitatives ont fortement influé sur la "fécondance du sperme". En effet la vitalité et la mobilité des spermatozoïdes sont universellement reconnues comme des facteurs essentiels de la fertilité masculine. Dans les couples, les grossesses sont d'autant moins fréquentes que le pourcentage d'altérations cinétiques des spermatozoïdes est élevé [10].

La tératozoospermie, associée aux autres anomalies 
Tableau 1 : Pathologies répertoriées à l'examen clinique des patients.

\begin{tabular}{lcc}
\hline Pathologies & $\begin{array}{c}\text { Nombre } \\
\text { de cas }\end{array}$ & Pourcentage \\
\hline Testicule non descendu & 140 & 0,8 \\
Hypo ou atrophie testiculaire & 122 & 0,3 \\
Torsion du cordon spermatique & 53 & 0,7 \\
Infections génitales & 108 & 0,6 \\
Varicocèle & 1764 & 10 \\
\hline
\end{tabular}

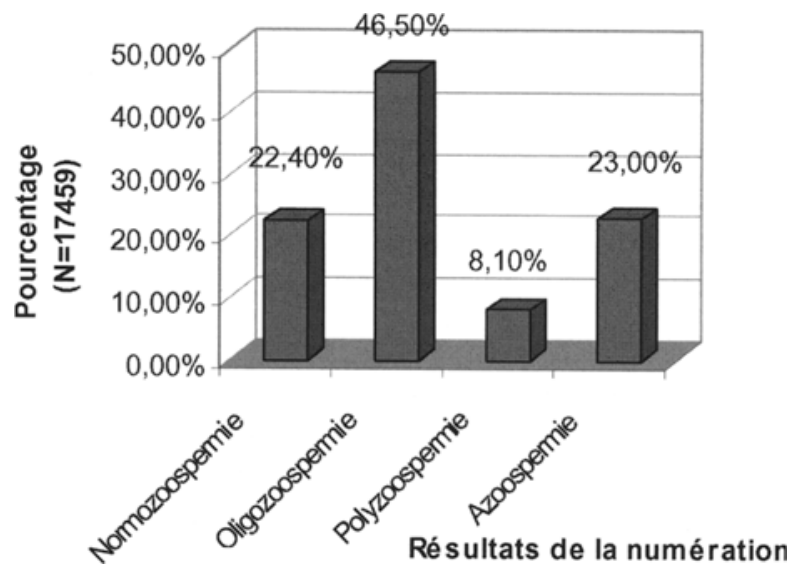

Figure 2 : Répartition des patients selon la numération des spermatozoïdes.

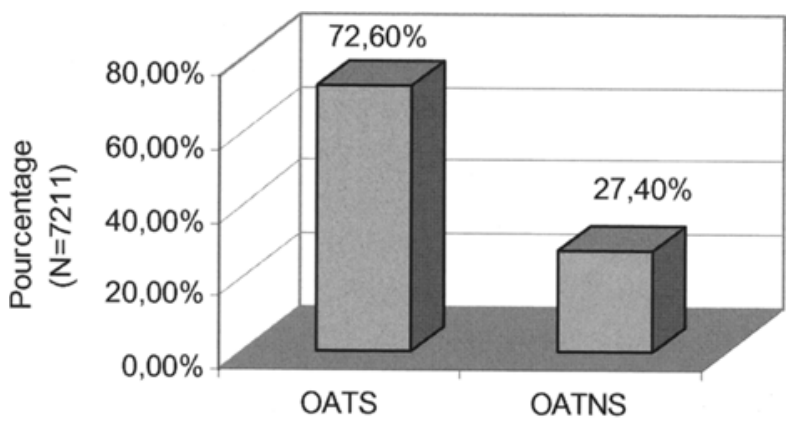

Anomalies associées

Figure 4 : Répartition des anomalies (quantitatives et qualitatives) associées.

OATS : oligo-asthéno-tératozoospermie

OATNS : oligo-asthéno-térato-nécrozoospermie.

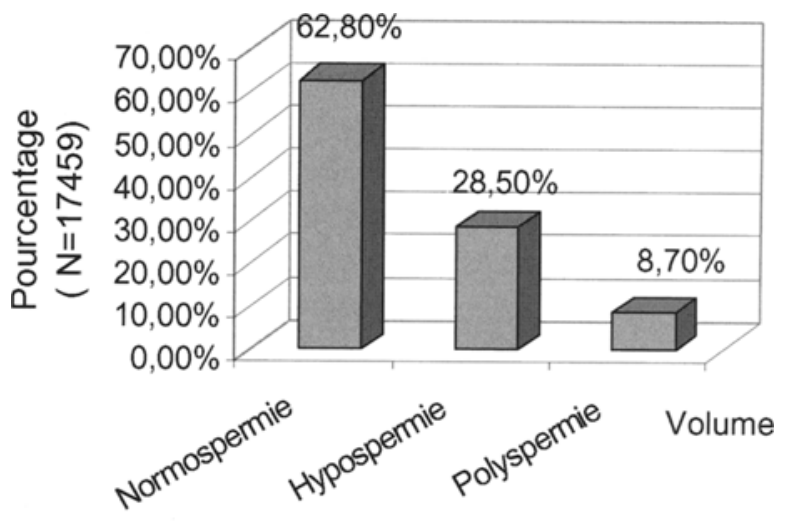

Répartition selon le volume du prélèvement

Figure 1 : Répartition des patients selon le volume du prélèvement.

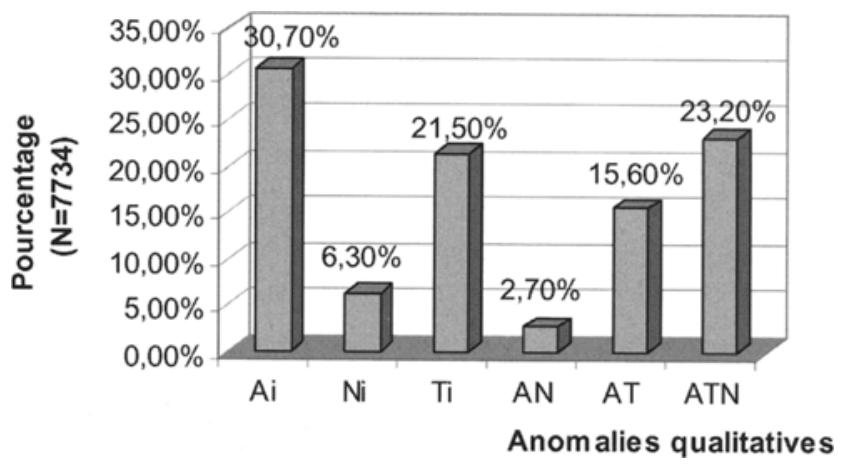

Figure 3 : Répartition des anomalies qualitatives.

$A i=$ Asthénozoospermie isolée

AN = Asthéno-nécrozoospermie

$\mathrm{Ni}=$ Nécrozoospermie isolée

$A T=$ Asthéno-tératozoospermie

$T i=$ Tératozoospermie isolée

ATN = Asthéno-térato-nécrozoospermie. 
spermiologiques prend des proportions importantes dans notre étude, confirmant ainsi l'idée selon laquelle les oligo-asthéno-tératozoospermies

(OATS) représentaient $60 \%$ des causes d'infertilité dans la population générale [12]. Le caractère spécifique de certaines de ces oligo-asthéno-tératozoospermies avec un profil spermiologique de varicocèle conforte les résultats des travaux de Abdelrahim et al. [1] qui ont rapporté une diminution de la spermatogenèse, une desquamation des gamètes immatures dans la lumière tubulaire et un épaississement de la paroi des tubes chez des porteurs de varicocèle. Guèye et al. [6] quant à eux rapportaient l'influence positive de la cure chirurgicale de varicocèle sur la qualité du sperme.

L'importance des dystrophies réactionnelles avec une spermoculture positive confirmerait le rôle non négligeable des processus inflammatoires et/ou infectieux dans l'infertilité masculine en Afrique bien que les troubles endocriniens, malformatifs et génétiques semblent prédominés.

\section{CONCLUSION}

Le problème de l'infertilité du couple en Afrique noire reste entier. La femme est presque toujours indexée et pourtant la part de responsabilité masculine est bien réelle.

Le profil spermiologique de l'époux dans ces couples infertiles est caractérisé par l'importance des altérations polymorphes comme l'oligo-asthénotérato-nécrozoospermie et de l'azoospermie sécrétoire.

L'idéal serait la mise en place de structures multidisciplinaires réunissant des cliniciens, des biologistes et des spécialistes de la procréation médicalement assistée pour permettre de faire face aux multiples problèmes posés par l'infertilité en milieu négro-africain.

\section{REFERENCES}

1. ABDELRAHIM F., MOSTAFA A., HAMDY A., MABROUK M., EL KHOLY M., HASSAN O. : Testicular morphology and function in varicocele patient. Pre-operative and post-operative histopathology. Br. J. Urol., 1993, $72: 643-647$.

2. AFOUTOU J.M., DIALLO A.S., D'ALMEIDA C. et al. : Place du test post coïtal de Hünher dans le bilan de la stérilité conjugale en milieu africain au Sénégal. Bull. Soc. Path. Ex., 1997, $90: 120-123$.

3. ORGANISATION MONDIALE DE LASANTE : Manuel pour l'analyse du sperme humain et de l'interaction des spermatozoïdes avec le mucus cervical. Paris, Editions INSERM, 1993.

4. DAVID G., BISSON J.P., CZYGLIK F, JOUANNET P., GERNIGNON C. : Anomalies morphologiques du spermatozoïde humain. J. Gynecol. Obstét. Reprod., 1975, 4 : 17-36.

5. GUERIN J.F. : Le sperme normal. Rev. Prat., 1993, 43 : 932-935
6. GUEYE S.M., FALL P., NDOYE A.K. et al. : Influence de la cure de varicocèle sur la qualité du sperme. Andrologie, 1999, $9: 376-379$.

7. GUIRAUD J.R., BREMONDA., ROTTEN D. : Abrégé de gynécologie. Masson, Paris, 1984 : 227-232.

8. IKECHEBELU J.I., ADINMA J.I., ORIE E.F., IKEGWUONU S.O. : High prevalence of male infertility in south-eastern Nigeria. J. Obstet. Gynaecol., 2003, 23 : 657-659.

9. KITILLAT. : Semen quality of suspected infertile Ethiopians at Family Guidance association of Ethiopia (FGAE) Central Clinic, Addis Ababa: a retrospective review. Ethiop. Med. J., 2002, $40: 325-334$.

10. MAC LEOD J.: Seminal cytology in the presence of varicocele. Fertil. Steril., 1965, $16: 735$.

11. MERINO G., CARRANZA-LIRA S. : Semen characteristics, endocrine profiles, and testicular biopsies of infertile men of different ages. Arch. Androl., 1995, $35:$ 219-224.

12. PONTONNIER F., BUJAN L. : Comment reconnaître et classer une infécondité masculine. Rev. Prat. Paris, 1993, 43 : 941-947.

Manuscrit reçu : mars 2006 ; accepté mai 2006.

\section{ABSTRACT}

Semen analysis in infertile couples in Senegal

Babacar DIAO, Oumar FAYE, Papa Ahmed FALL, Abdoulaye Séga DIALLO, Alain Khassim NDOYE, José Marie AFOUTOU

Objective: To define the role of male infertility in black African couples in Senegal and to establish the semen profile.

Material and Methods: We analysed 17,459 sperm counts and 5,563 post-coital tests from patients consulting for primary or secondary infertility between January 1982 and December 2002. Negative and deficient sperm counts of post-coital tests were studied to demonstrate the responsibility of male infertility. For sperm counts, we studied the patient's age, the mode of semen collection, the volume of ejaculate and semen characteristics.

Results: Primary sterility (68.4\%) was twice as frequent as secondary sterility (31.6\%). Male infertility (31.7\%) was twice as frequent as female infertility $(14.7 \%)$. Twenty eight per cent of patients presented hypospermia. Isolated oligozoospermia was observed in $10 \%$ of cases and azoospermia was detected in $23 \%$ of cases. Qualitative sperm changes were observed in $44.3 \%$ of cases.

A positive semen culture was reported in $21.3 \%$ of cases. Combinations (qualitative sperm changes and abnormalities of number) were observed in $43.4 \%$ of cases. Oligo-astheno-teratozoospermia (OATS) with spermatozoa with an elongated head, cytoplasmic 
remnants and angulation, characteristic of varicocele was significantly more frequent in patients with varicocele $(97 \%$ of men with right varicocele and $98.2 \%$ of men with bilateral varicocele). Reactive epididymosemino-prostatic dystrophy was observed in $11.9 \%$ of cases

Conclusion: Male infertility plays a real role. The semen profile of the husband of a sterile couple in Senegal is characterized by the importance of polymorphic alterations such as oligo-astheno-teratospermia and secretory azoospermia.

Key words: male infertility, oligo-astheno-teratozoospermia, secretory azoospermia 